\title{
Selected Papers of the Fourth International Conference on the Theory and Practice of Natural Computing, TPNC 2015
}

\author{
Adrian-Horia Dediu ${ }^{1}$ Carlos Martín-Vide ${ }^{2}$
}

Published online: 29 December 2017

○) Springer-Verlag GmbH Germany, part of Springer Nature 2017

This special issue of the journal "Soft Computing-A Fusion of Foundations, Methodologies and Applications" offers extended versions of some of the best papers presented at the Fourth International Conference on the Theory and Practice of Natural Computing, TPNC 2015, held in Mieres, Spain, on December 15-16, 2015. The conference was organized by the European Centre for Soft Computing in Mieres and the Research Group on Mathematical Linguistics (GRLMC) from Rovira i Virgili University, Tarragona, Spain.

TPNC 2015 was the fourth event in a series dedicated to hosting and promoting research in a wide spectrum of computational models, methods and techniques inspired by information processing in nature. We encourage both theoretical contributions in soft computing, computing architectures, and formal models as well as solutions for practical problems based on natural computing methods.

Out of 30 submissions to the conference, 12 papers were accepted (which represents a competitive acceptance rate of about $40 \%$ ). Among them, the authors of 4 papers were invited to submit to this special issue. Each submission was reviewed by two or three experts and, on the basis of their comments, the guest editors decided to accept 2 papers for this special issue (which represents an acceptance rate of about $7 \%$ out of the submissions to the conference).

Next, we present the papers included in our special issue sorted by the first author name. For each paper we mention the most important results.

Patricia Conde-Clemente, José M. Alonso, and Gracián Triviño, in their paper Towards Automatic Generation of Lin-

Adrian-Horia Dediu

adrian-horia.dediu@superdata.ro

1 SuperData, Bucharest, Romania

2 Research Group on Mathematical Linguistics (GRLMC), Rovira i Virgili University, Av. Catalunya, 35, 43002

Tarragona, Spain guistic Advice for Saving Energy at Home, describe a new architecture able to generate customized linguistic descriptions of data in the realm of energy consumption/saving. The aim of the descriptions is to provide saving advices to householders regarding their domestic consumption. The most relevant contribution of the paper, in terms of what has been proposed in the literature about fuzzy linguistic descriptions or summaries of data, is the inclusion of user profiles that allow to adapt the generated reports to different kinds of target users, and the inclusion of the discourse history to avoid repetition of information previously communicated.

In their paper Card-Based Protocols Using Unequal Division Shuffles, Akihiro Nishimura, Takuya Nishida, Yu-ichi Hayashi, Takaaki Mizuki, and Hideaki Sone aim to analyze and make use of an uncommon shuffle operation, which has been previously proposed to achieve a reduction in the number of cards in committed AND protocols by one. The main result of the paper is a reduction in the number of cards for copy protocols by one, by doubling the average number of trials using this "unequal division shuffle." An implementation of the (double) unequal division shuffle by sliding cover boxes and envelopes of varying size confirms the theoretical results.

We thank all reviewers for their valuable work. We also thank all the authors for their contributions as well as the editorial team of the journal for their professional support and collaboration.

\section{Compliance with ethical standards}

Conflict of interest None of the authors have any financial interest in relation to this study or its results. 